\title{
Comparing endoscopic intervention against fully covered self-expanding metal stent placement for post-endoscopic sphincterotomy bleed (CEASE Study)
}

Authors

Institutions
Justin Cochrane ${ }^{1}$, Greg Schlepp ${ }^{2}$

${ }^{1}$ Providence Sacred Heart - Internal Medicine Residency, Spokane, Washington, United States

${ }^{2}$ Spokane Digestive Disease Center - Gastroenterology, Spokane, Washington, United States submitted 3. February 2016 accepted after revision 9. September 2016

\section{Bibliography}

Dol http://dx.doi.org/

10.1055/s-0042-118227

Published online: 24.11.2016

Endoscopy International Open 2016; 04: E1261-E1264

(c) Georg Thieme Verlag KG Stuttgart · New York

E-ISSN 2196-9736

Corresponding author Justin Cochrane Providence Sacred Heart Internal Medicine Residency Sacred Heart Medical Center PO box 2555

Spokane Washington 99220 United States

Phone: +1-509-474-3237

Fax: +1-509-744-3969

justincg5@yahoo.com
License terms

(이요
Background and study aims: Limited data exist for the use of fully covered self-expanding metal stent (FCSEMS) as an intervention for immediate bleeds post-endoscopic sphincterotomy (ES) after primary endoscopic intervention failure or to reduce the number of delayed bleeding events in patient with increased risk of bleeding post-ES. Patients and methods: We evaluated a retrospective cohort of individuals who had ES performed from 2011 to 2014. A total of 700 patients were identified with 67 patients having post-ES bleeding. The FCSEMS treatment group included 23 patients and the non-FCSEMS treatment group included 44 patients. The primary end point was rate of change of $\mathrm{Hgb}$ at 72 hours after ES in the FCSEMS group and the primary endoscopic intervention-only group. A comparison also was made between the FCSEMS and non-FCSEMS group with regards to proportion of coagulopathy and number of delayed bleeding events.

\section{Introduction \\ $\nabla$}

Endoscopic sphincterotomy (ES) was first utilized in 1974 in conjunction with endoscopic retrograde cholangiopancreatography (ERCP) for treatment of biliary and pancreatic diseases. An important complication of ES is development of post-ES bleeding, which carries an overall mortality rate of $1.5 \%$ [1]. Most bleeding with sphincterotomy arises near the apex in close association with, the pancreatic orifice and surrounding blood vessels [2]. Post-ES bleeding ranges in incidence from $1 \%$ to $2 \%$ to $10 \%$ to $48 \%$, depending on the definition of significant bleeding [3]. The decision to intervene for post-ES bleeding has been traditionally based upon an endoscopist's assessment of bleeding severity based on volume of blood (oozing vs pulsatile) and duration of bleeding. The typical care for "endoscopically significant" bleeding is through endoscopic intervention with balloon tamponade, sclerotherapy,
Results: The FCSEMS treatment group had a lower bleeding rate at 72 hours $(0.66 \mathrm{~g} / \mathrm{dL}$ vs $1.98 \mathrm{~g} / \mathrm{dL} P$ $<0.001)$, increased proportion of patients at high risk of bleeding ( $40 \%$ vs $9 \% P$ value 0.008 ), and increased frequency of bleeding events that were moderately severe ( $52 \%$ vs $9 \% P=0.0002)$ compared to the non-FCSEMS treatment group. The FCSEMS group included 9 patients at increased risk of bleeding and no patients with delayed bleed compared to the non-FCSEMS group, in which all 4 patients at increased risk of bleeding developed a delayed bleed.

Conclusion: FCSEMS can provide homeostasis after primary endoscopic intervention failure, thus reducing the need for high-risk procedures. FCSEMS can reduce delayed bleeding events in patients at high risk of post-ES bleeding.

epinephrine injection, thermal cautery, or clips [4-9].

Over the years the use of fully covered self-expanding metal stents (FCSEMS Wallstent, Boston Scientific) on our unit has served as a means to control hemorrhage in post-ES bleeding after primary endoscopic intervention failure and thus provided a less invasive treatment option compared to surgery or arterial embolization. This study, therefore, was conducted to retrospectively analyze utility of FCSEMS in the setting of post-ES bleeding. The primary objective was to asses bleeding severity as expressed in changes of $\mathrm{Hgb}$ at 72 hours and the development of delayed bleeding in patients at high risk of bleeding between non-FCSEMS and FCSEMS treatment groups. 
Table 1 Characteristics of FCSEMS treatment group.

\begin{tabular}{|c|c|c|c|c|c|c|}
\hline Age & Sex & Severity of bleeding & Indication for ES & Primary endoscopic Intervention & Transfusion & FCSEMS Size $\mathrm{mm} \times \mathbf{m m}$ \\
\hline 73 & $\mathrm{~F}$ & Mild & Cholelithiasis & Balloon Tamponade & $\mathrm{N}$ & $10 \times 40$ \\
\hline 71 & $\mathrm{~F}$ & Moderate & BBS & Balloon Tamponade & $\mathrm{N}$ & $10 \times 40$ \\
\hline 58 & $\mathrm{~F}$ & Mild & Cholelithiasis & Balloon Tamponade & N & $10 \times 40$ \\
\hline 85 & $\mathrm{~F}$ & Mild & Cholelithiasis & Balloon Tamponade & N & $10 \times 60$ \\
\hline 92 & $\mathrm{~F}$ & Moderate & Cholelithiasis & Balloon Tamponade & N & $10 \times 40$ \\
\hline 71 & M & Mild & BBS & Balloon Tamponade & N & $10 \times 40$ \\
\hline 85 & M & Moderate & Cholelithiasis & Balloon Tamponade & $\mathrm{N}$ & $10 \times 40$ \\
\hline 54 & M & Moderate & Cholelithiasis & Balloon Tamponade & N & $10 \times 40$ \\
\hline 65 & $\mathrm{~F}$ & Mild & Sphincter Oddi dysfunction & Balloon Tamponade & $\mathrm{N}$ & $10 \times 40$ \\
\hline 52 & $\mathrm{~F}$ & Moderate & Cholelithiasis & Balloon Tamponade & N & $10 \times 60$ \\
\hline 76 & M & Moderate & Pancreatic mass & Balloon Tamponade & $\mathrm{N}$ & $10 \times 40$ \\
\hline 50 & M & Mild & Cholelithiasis & Balloon Tamponade & N & $10 \times 40$ \\
\hline 63 & $\mathrm{~F}$ & Moderate & Pancreatic mass & Balloon Tamponade & $\mathrm{N}$ & $10 \times 40$ \\
\hline 82 & M & Mild & BBS & Balloon Tamponade & $\mathrm{N}$ & $10 \times 60$ \\
\hline 89 & $\mathrm{M}$ & Mild & Cholelithiasis & Balloon Tamponade & N & $10 \times 40$ \\
\hline 50 & M & Moderate & Cholelithiasis & Balloon Tamponade & N & $10 \times 60$ \\
\hline 44 & $M$ & Moderate & BBS & Epinephrine & $\mathrm{N}$ & $10 \times 80$ \\
\hline 90 & $\mathrm{~F}$ & Moderate & Cholelithiasis & Epinephrine & Y & $10 \times 60$ \\
\hline 53 & M & Severe & ES & Clips \& EPI & N & $10 \times 40$ \\
\hline 64 & M & Moderate & Cholelithiasis & Epinephrine & N & $10 \times 40$ \\
\hline 71 & M & Mild & Cholelithiasis & Balloon Tamponade & N & $10 \times 40$ \\
\hline 72 & M & Mild & Cholelithiasis & Balloon Tamponade & N & $10 \times 40$ \\
\hline 64 & M & Moderate & Cholelithiasis & Epinephrine & $\mathrm{N}$ & $10 \times 40$ \\
\hline
\end{tabular}

BBS, benign biliary stricture; EPI, epinephrine; FCSEMS, fully covered self-expanding metal stent

\section{Patients and methods}

We reviewed data from 700 adults (>18 years) who received an endoscopic sphincterotomy during a 3-year time period (2011 to 2014). These patients were retrospectively evaluated utilizing electronic medical records (Meditech, Epic), at a tertiary referral center, Providence Sacred Heart Medical Center. Inclusion criteria consisted of developing an immediate bleed or delayed bleed after endoscopic sphincterotomy. Patients were excluded if they had no initial hemoglobin prior to ES, no follow up hemoglobin at 72 hours after ES, or developed bleeding at other sites after sphincterotomy performed. The Institutional Review Board associated with Providence Sacred Heart Medical Center approved the collection and review of data at their institution for the purpose of this study by the primary author.

Post-ES bleeding was determined by searching the key terms oozing, bleeding, or hemorrhage in gastroenterologist procedure notes. Indications for endoscopic sphincterotomy and distributions of treatment groups refer to $\bullet$ Table1 and $\bullet$ Fig. 1. Severity of bleeding was based on description of bleeding by the performing endoscopist for mild (oozing) and moderate bleeding (pulsatile) and duration of bleeding greater than 2 to 3 minutes. Severe bleeding was determined by change in $\mathrm{Hgb}>4 \mathrm{~g} / \mathrm{dL}$ at 72 hours or transfusion received. Failure of primary endoscopic intervention and decision for FCSEMS treatment was at the discretion of the endoscopist performing sphincterotomy. Primary endoscopic intervention included sclerotherapy, balloon tamponade, hemoclip placement or epinephrine injection. Blood bank records at the time of post-ES bleeding were reviewed to determine if transfusions were given and the number of units received.

Determination of the patient population at high risk for bleeding post-ES was derived from the Freeman et al [10] study on ES complications. Criteria included coagulopathy defined as platelets less than $50 \times 10^{9} / \mathrm{L}$, INR $\geq 1.3$, cirrhosis child class $\mathrm{C}$, or expo-

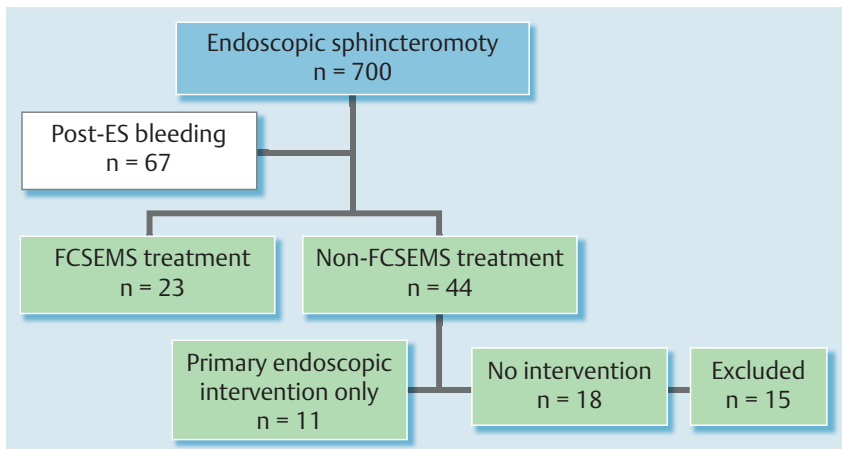

Fig. 1 Distribution of patient population. ES, endoscopic sphincterotomy; FCSEMS, fully covered self-expanding metal stent.

sure to anticoagulation medication within 72 hours of sphincterotomy. All patients were followed up to 30 days post-ES bleeding event with chart review for identification of delayed bleeding manifestations, hematochezia, melena or repeat EGD/ERCP.

\section{Endoscopic sphincterotomy procedure}

All endoscopic procedures were performed in the endoscopy suite. Conscious sedation (fentanyl, midazolam) or monitored anesthesia was administered at the discretion of the endoscopist or consulting anesthesiologist. Endoscopic retrograde cholangiopancreatography was performed with Olympus Duodenoscope TJF-160VR (Olympus America Inc, USA). Standard sphincterotomy was performed with $20 \mathrm{~mm}$ to $30 \mathrm{~mm}$ cutting wire length or needle knife. Electrocautery with ERBE VIO units (ERBE USA inc, Marietta GA) setting of ENDO cut 1 cutting effect 2, cutting duration 3, cutting interval 3, and max watts of 200 amps. All ES procedures were performed by 2 experienced pancreaticobiliary endoscopist. 
Table 2 Comparison between treatment groups.

\begin{tabular}{|c|c|c|c|}
\hline & FCSEMS & Non-FCSEMS & $P$ value \\
\hline Age (Average years) & $64.4 \pm 14.5(\mathrm{Cl} 5.93)$ & $63.9 \pm 16.7(\mathrm{Cl} 4.93)$ & 0.06 \\
\hline Sex Male \% & 61 & 43 & $>0.05$ \\
\hline Platelet (SI units $10^{9} / \mathrm{L}$ ) & $191 \pm 83(\mathrm{Cl} 33.9)$ & $179 \pm 89(\mathrm{Cl} 26.5)$ & 0.2 \\
\hline Change $\mathrm{Hgb}(\mathrm{g} / \mathrm{dL})$ & $0.69 \pm 0.88(\mathrm{Cl} 0.36)$ & $1.56 \pm 1.29(\mathrm{Cl} 0.48)$ & 0.009 \\
\hline Mild bleeding \% & 43 & 85 & 0.0003 \\
\hline Moderate bleeding \% & 52 & 9 & 0.0002 \\
\hline Severe bleeding \% & 4 & 2 & $>0.05$ \\
\hline Coagulopathy \% & 40 & 9 & 0.008 \\
\hline Transfusion \% & 4 & 4 & $>0.05$ \\
\hline Delayed bleed \% (in high risk bleeding population) & 0 & 100 & 0.001 \\
\hline
\end{tabular}

FCSEMS, fully covered self-expanding metal stent; Hgb, hemoglobin; $\mathrm{Cl}$, confidence Interval

\section{Statistical analysis}

Continuous variables were expressed as a mean \pm standard deviation and categorical data as $\mathrm{N}(\%)$. A 2-tailed $\mathrm{T}$ test was performed to determine significance of the means between groups and Pearson chi-squared test or Fischer exact test, when appropriate, was used to compare categorical data between groups with a $P$ value $<0.05$ being significant. Confidence intervals are reported at $95 \%$.

\section{Results}

$\nabla$

A total of 700 patients were identified as having an ES from 2011 to 2014. Of them, 67 patients developed post-ES bleeding, for an incidence of $1 \%$. $\odot$ Fig. 1 shows the distribution of the patient population. The FCSEMS treatment group demonstrated a decreased hemoglobin change at 72 hours $(0.69 \mathrm{~g} / \mathrm{dL}$ vs $1.56 \mathrm{~g} / \mathrm{dL} P$ value $=0.009$ ) despite having a higher proportion of moderate bleeding episodes ( $52 \%$ vs $9 \% P=0.002$ ). The proportion of patients determined at high risk for post-ES bleeding was higher in the FCSEMS treatment group ( $40 \%$ vs $9 \% P=0.008$ ), but they had fewer delayed bleeding episodes than the patients in the nonFCSEMS treatment group ( $0 \%$ vs $100 \% P=0.001$ ). The patients in the non-FCSEMS treatment group who developed a delayed bleed had significantly greater change in hemoglobin at 72 hours $(4.15 \mathrm{~g} / \mathrm{dL})$, as shown in $\odot$ Table 2 .

Analysis of Hgb change at 72 hours was further compared between the FCSEMS group and non-FCSEMS group divided into primary endoscopic intervention and no intervention. Patients with no intervention developed the highest rate of change at 1.8 $\mathrm{g} / \mathrm{dL}$. A direct comparison between FCSEMS treatment group and those receiving primary endoscopic intervention demonstrated significantly less hemoglobin change at 72 hours $(0.69 \mathrm{~g} / \mathrm{dL}$ vs $1.42 \mathrm{~g} / \mathrm{dL} ; P=0.04$ ).

No adverse events were noted with FCSEMS insertion, during occupancy, or removal. Average duration of stent placement was 88 days.

\section{Discussion}

$\nabla$

These data are the first to examine FCSEMS in post-ES bleeding after primary endoscopic intervention failure, and in patients at high risk of developing a delayed bleed. This suggests a clinically important utility for FCSEMS in the post-ES bleeding algorithm. In 2007, Ferreira and Baron [3] proposed an algorithm for treat- ment of immediate post-ES bleeding that calls for identification of endoscopically significant bleeding before instituting primary endoscopic intervention, followed by monotherapy with balloon tamponade, epinephrine injections, thermal cautery, or clips. If continued bleeding is evident, combination therapy should be used. Failure of endoscopic therapy necessitates arterial embolization or surgery. Limited data exist for interventions that bridge the gap between primary endoscopic intervention and more invasive approaches with surgery or arterial embolization. In addition, this algorithm does not include a strategy for patients at increased risk of developing a delayed bleed. We propose a treatment algorithm where FCSEMS is the treatment of choice after primary endoscopic intervention failure, or as primary intervention in patients at high risk of continued bleeding post-procedure (० Fig.2).

Two retrospective case series evaluated FCSEMS as a treatment after primary endoscopic failure. No studies have explored the use of FCSEMS as primary intervention for prevention of delayed bleeds in patients at high risk of continued bleeding post-ES. Itoi ET el [11] and Shah ET el [12] achieved 100\% homeostasis in 5 patients with immediate bleeding post-ES after primary endoscopic intervention failure. A total of 10 patients were identified in these studies as being at high risk of bleeding, 9 of whom (90\%) developed a delayed bleed requiring transfusions and repeat endoscopic procedures to control post-ES bleeding. The 1 patient who received a FCSEMS as primary intervention had bleeding controlled and required no transfusion or repeat endoscopic procedures.

We demonstrated that the FCSEMS treatment group achieved homeostasis in 23 patients (100\%) after primary endoscopic intervention failure. The FCSEMS treatment group had a lower $\mathrm{Hgb}$ change at 72 hours in a patient population with significantly higher severity of post-ES bleeding. The FCSEMS treatment group had no delayed bleeding events in 9 patients, compared to 4 delayed bleeding events in the primary endoscopic intervention treatment group in patients at high risk of bleeding.

To our knowledge, our study is the largest cohort evaluation of FCSEMS to provide hemostasis in post-ES bleeding after primary endoscopic intervention failure. Also, these are the first data to compare the efficacy of FCSEMS to primary endoscopic intervention for prevention of delayed bleeds in patients at increased risk of bleeding.

Major concerns about utilizing FCSEMS for post-ES bleeding after primary endoscopic intervention failure are a potential increase in the rate of pancreatitis from pancreatic duct obstruction and increased cost. Our cohort population had no adverse events of 


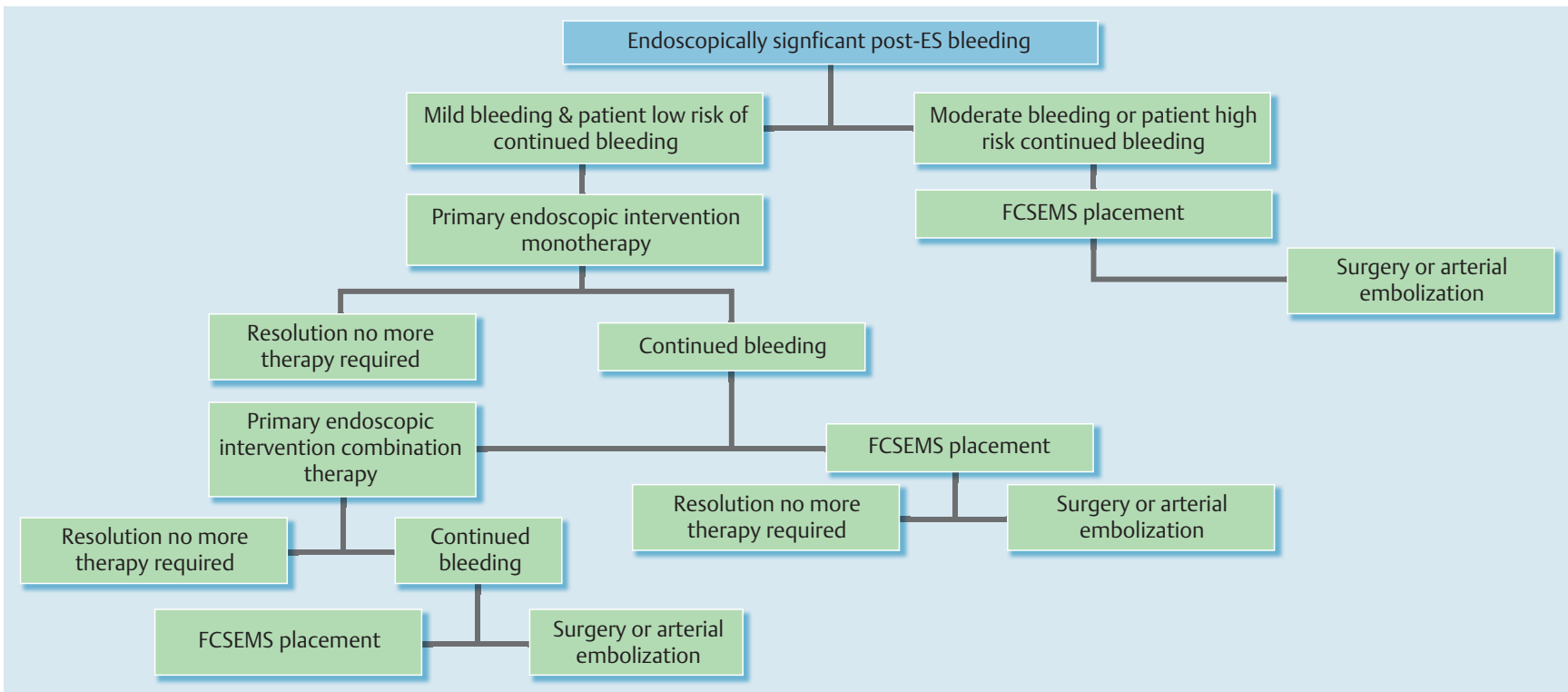

Fig. 2 Proposed treatment algorithm for post-ES bleeding, ES, endoscopic sphincterotomy; FCSEMS, fully covered self-expanding metal stent.

pancreatitis and pancreatic duct stents were not utilized for prevention of pancreatic duct obstruction. However, given the limited size of our cohort, further evaluation is warranted on pancreatitis secondary to FCSEMS placement and the need for pancreatic stents. FCSEMS themselves incur an extra cost besides the need for more procedures. We justify the extra cost by hypothesizing the likely reduction in the number of blood products needed, reduced length of stay in the hospital, and prevention of surgical intervention. Cost-effectiveness analysis was not performed as a part of this study and further study is warranted.

Several limitations of the study should be considered. First, selection bias is inherent in a retrospective cohort. Second, data on subsequent follow up was lacking in multiple patients in the non-FCSEMS arm. Third, all patients in the FCSEMS arm received primary endoscopic intervention prior to stent placement, which may have had an impact on reducing the post-ES bleeding rate. Finally, several patients had prolonged duration of FCSEMS in place, thus limiting our ability to make a statement about appropriate timing of stent removal; further studies are need to determine adequate duration for treatment. A formal randomized controlled trial is warranted.

\section{Conclusion}

$\nabla$

Given the best current data, FCSEMS appears to provide hemostasis after primary endoscopic intervention failure, thus reducing the need for arterial embolization or surgery. Our study supports using FCSEMS in patients with increased severity of immediate bleeding or who are at increased risk of delayed bleeding after ES.

\section{References}

1 Cotton PB, Lehman G, Vennes J et al. Endoscopic sphincterotomy complications and their management: an attempt at consensus. Gastrointest Endosc 1991; 37: 383-393

2 Jamidar PA. MD. FACG. FASGE. Nov. 22, 2013 Endoscopic sphincterotomy. Medscape Reference article. Retrieved July, 2015, http://emedicine.medscape.com/article/1891681-overview

3 Ferreira LE, Baron TH. Post-sphincterotomy bleeding: who, what, when, and how. Am J Gastroenterol 2007; 102: 2850-2858

4 Leung JWC, Chan FKL, Sung JJY et al. Endoscopic sphincterotomy-induced hemorrhage: a study of risk factors and the role of epinephrine injection. Gastrointest Endosc 1995; 42: 550-554

5 Vusconez C, Llach J, Bordas JM et al. Injection treatment of hemorrhage induced endoscopic sphincterotomy. Endoscopy 1998; 30: 37-39

6 Sherman S, Hawes RH, Nisi R et al. Endoscopic sphincterotomy-induced hemorrhage: treatment with multipolar electrocoagulation. Gastrointest Endosc 1992; 38: 123-126

7 Matsushita M, Takakuwa H, Nishio A. Efficacy of endoscopic hemostatic injection orad to the site of post-sphincterotomy bleeding: anatomic confirmation. Gastrointest Endosc 2001; 53: 270

8 So YH, Choi YH, Chung JW et al. Selective embolization for post-endoscopic sphincterotomy bleeding: technical aspects and clinical efficacy. Korean J Radiol 2012; 13: 73-81

9 Goodall RJ. Bleeding after endoscopic sphincterotomy. Ann R Coll Surg Engl 1985; 67: 87

10 Freeman ML, Nelson DB, Sherman $S$ et al. Complications of endoscopic biliary sphincterotomy. N Engl J Med 1996; 335: 909-918

11 Itoi T, Yasuda I, Doi S et al. Endoscopic hemostasis using covered metallic stent placement for uncontrolled post-endoscopic sphincterotomy bleeding. Endoscopy 2011; 43: 369-372

12 Shah JN, Marson F, Binmoeller KF. Temporary self-expandable metal stent placement for treatment of post-sphincterotomy bleeding. Gastrointest Endosc 2010; 72: 1274-1278

\section{Competing interests: None}

